Network Working Group

C. Malamud

Request for Comments: 4095

Memory Palace Press

Category: Standards Track

May 2005

\title{
Attaching Meaning to Solicitation Class Keywords
}

Status of This Memo

This document specifies an Internet standards track protocol for the Internet community, and requests discussion and suggestions for improvements. Please refer to the current edition of the "Internet Official Protocol standards" (STD 1) for the standardization state and status of this protocol. Distribution of this memo is unlimited.

Copyright Notice

Copyright (C) The Internet Society (2005).

Abstract

This document proposes a mechanism for finding a URI associated with a solicitation class keyword, which is defined in RFC 3865, the No Soliciting SMTP Service Extension. Solicitation class keywords are simple labels consisting of a domain name that has been reversed, such as "org.example.adv". These solicitation class keywords are inserted in selected header fields or used in the ESMTP service extension, including a new "No-Solicit:" header, which can contain one or more solicitation class keywords inserted by the sender.

This document specifies an application based on the Dynamic Delegation Discovery System (DDDS) described in RFC 3401 and related documents. An algorithm is specified to associate a solicitation class keyword with a URI which contains further information about the meaning and usage of that solicitation class keyword. For example, the registrant of the "example.org" domain could use this mechanism to create a URI which contains detailed information about the "org.example.adv" solicitation class keyword. 
Table of Contents

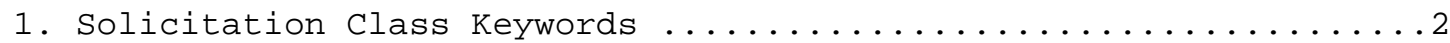

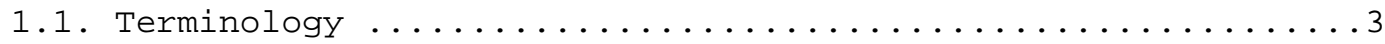

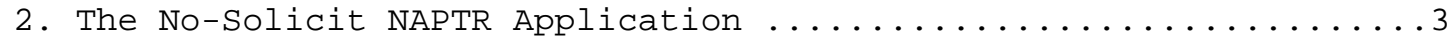

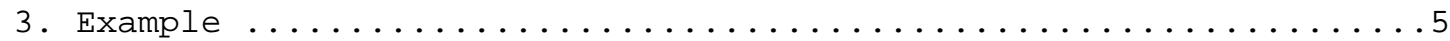

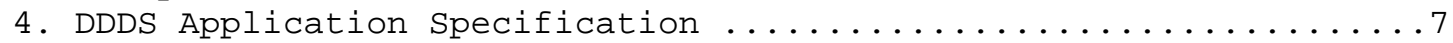

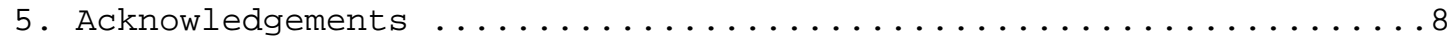

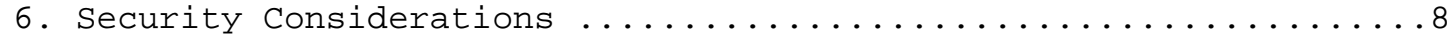

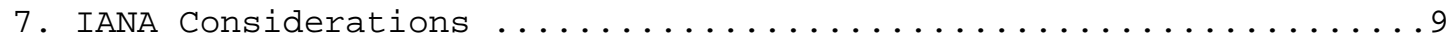

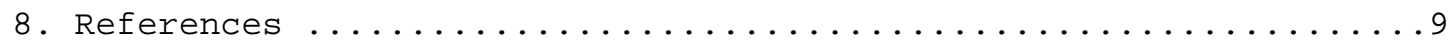

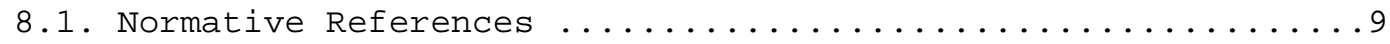

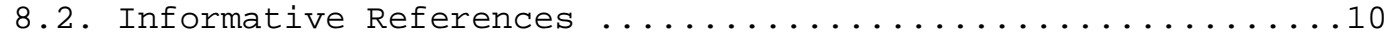

1. Solicitation Class Keywords

[RFC3865] defines the concept of a "solicitation class keyword", which is an arbitrary string or label which can be associated with an electronic mail message and transported by the ESMTP mail service as defined in [RFC2821] and related documents. Solicitation class keywords are formatted like domain names, but reversed. For example, the zone administrator of "example.com" might specify a particular solicitation class keyword such as "com.example.adv" that could be inserted in a "No-Solicit:" header by the message sender or in a trace field by a message transfer agent (MTA). This solicitation class keyword is inserted by the sender of the message, who may also insert a variety of other solicitation class keywords as defined by the sender or by other parties.

[RFC3865] explicitly places discovery of the meaning of a solicitation class keyword as outside of the scope of the basic ESMTP service extension. For the purposes of message transport, these solicitation class keywords are opaque. However, if RFC 3865 becomes widely used, a mail message might contain a large number of solicitation class keywords. The "No-Solicit:" header has keywords inserted by the sender of the message, which might include the sender's own keywords, as well as those mandated by regulatory authorities or recommended by voluntary industry associations. Likewise, the "received:" trace fields might contain a large number of keywords produced by message transfer agents, filtering software, forwarding software in the message user agent (MUA), or any other system in the chain of delivery.

As the number of keywords employed grows, it will be important to find a method for discovering the meaning behind the various solicitation class keywords. This document specifies such a mechanism, associating a solicitation class keyword with a URI which contains further information by using the DNS NAPTR Resource Record, 
which is defined in [RFC3403]. An explicit design goal is to keep the system as simple as possible. Approaches such as defining an XML-based structure that would contain specific meta-data about the solicitation class keyword or other approaches that define the format of the explanation were ruled out. Instead, the goal is to simply to associate a solicitation class keyword with a URI, which in turn contains an explanation of the keyword.

\subsection{Terminology}

The key words "MUST", "MUST NOT", "REQUIRED", "SHALL", "SHALL NOT", "SHOULD", "SHOULD NOT", "RECOMMENDED", "MAY", and "OPTIONAL" in this document are to be interpreted as described in BCP 14, [RFC2119].

\section{The No-Solicit NAPTR Application}

The DDDS framework of [RFC3401] and related documents provides a powerful set of mechanisms that can yield sophisticated applications such as ENUM as specified in [RFC3761]. There is a simplification of the DDDS framework called the Straightforward-NAPTR (S-NAPTR) application as specified in [RFC3958]. Unfortunately, S-NAPTR does not permit the use of the "U" flag for terminal lookups and does not support the regular expression field of the NAPTR RR. Since a replacement field in a NAPTR record must contain only a domain name, and our goal is to find a URI, this document does not use the S-NAPTR mechanism.

This document uses the NAPTR RR to do a single lookup from solicitation class keyword to URI. The character "." is first substituted for any instances of the character ":" and then the solicitation class keyword is reversed, using the character "." as the delimiter. This becomes the domain name lookup key. For example, "org.example:ADV" becomes "ADV.example.org".

Note On Domain Names: RFC3865 states that a solicitation class keyword consists of a valid domain name followed by the ":" character and by additional valid characters. Several points are important to remember for implementors. Since domain names are case insensitive and the ":" character is translated to the "." character, for purposes of this DDDS application, the following solicitation class keywords are syntactically equivalent: "com.example:ADV", "com.Example:adv", and "com:example:ADV".

In addition, it is important to remember that the resulting string must meet other DNS validity checks. In particular, domain labels are limited to 63 characters in length and the total length of the resulting string must be less than 253 characters. Any non-ASCII 
characters must be encoded using the Internationalized Domain Names (IDN) specifications in [RFC3490] and related documents. Note that non-ASCII characters may be encoded after the ":" character as well.

The fields of the NAPTR RR are used as follows:

- The "ORDER" and "PREFERENCE" fields are to be processed as specified in [RFC3403]: if multiple records are returned, the one (s) with the lowest "ORDER" value that have a matching "SERVICE" field MUST be used. Of those with the lowest ORDER value, those with the lowest "PREFERENCE" SHOULD be used.

- The "FLAGS" field MUST contain the character "U".

o The "SERVICES" field MUST contain only the string "no-solicit".

- The "REGEXP" field MUST contain a valid URI as further specified in this section.

o The "REPLACEMENT" field MUST be empty.

The "REGEXP" field is defined in [RFC3402] as consisting of a "delim-character", a POSIX Extended Regular Expression, another

"delim-character", a replacement value, and a final

"delim-character". For this application the following rules apply:

- The "delim-character" MAY be any valid character as defined in section 3.2 of [RFC3402].

- The extended regular expression MUST be empty.

- The replacement value MUST contain a valid URI as specified in [RFC3986].

- The replacement value SHOULD contain a URI limited to the "ftp", "http", and "https" schemes as specified in [RFC3986] and [RFC2660].

- The document that is retrieved at the URI SHOULD conform to [HTML-4.01], including the Accessibility Guidelines contained therein. 


\section{Example}

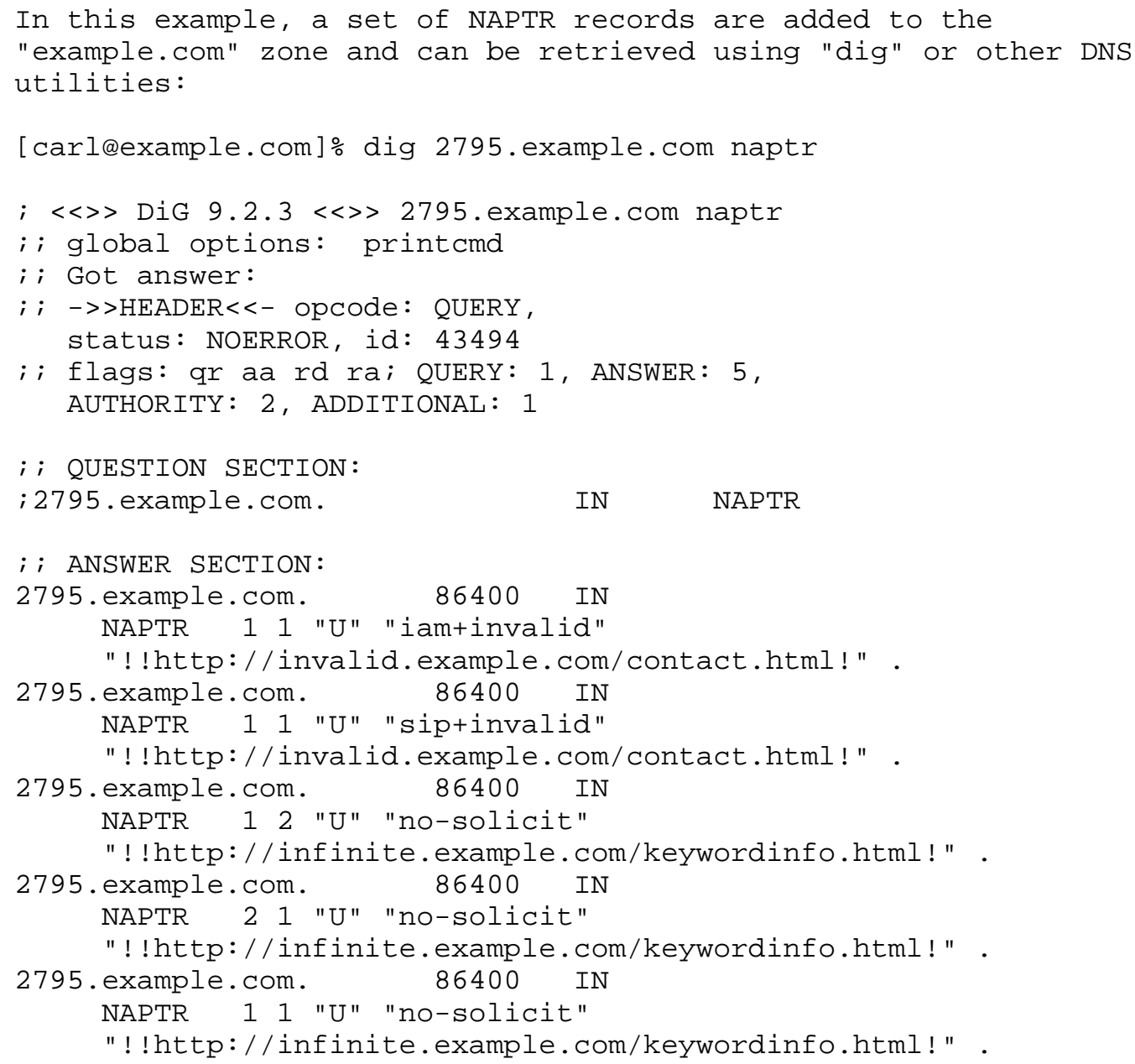




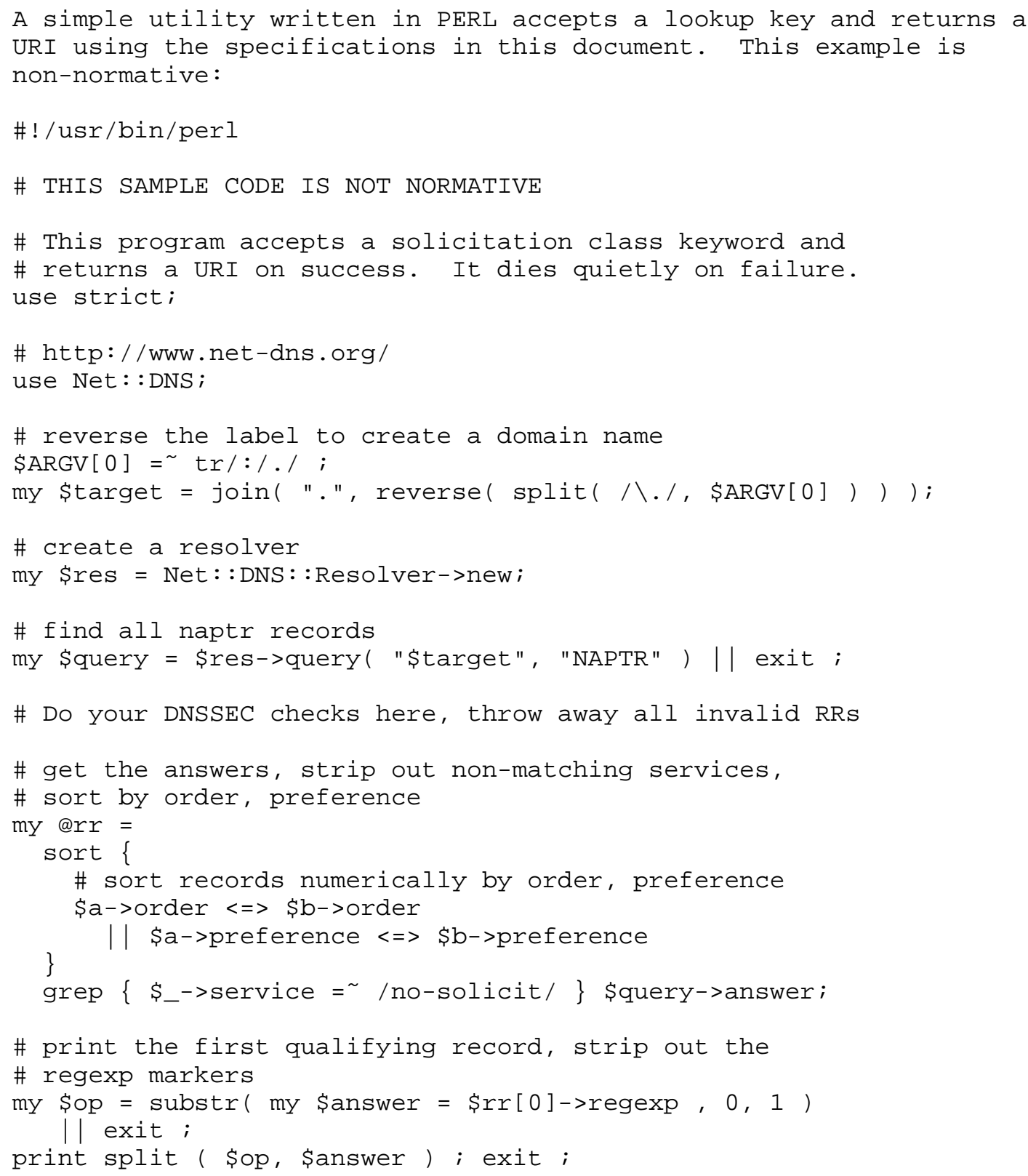




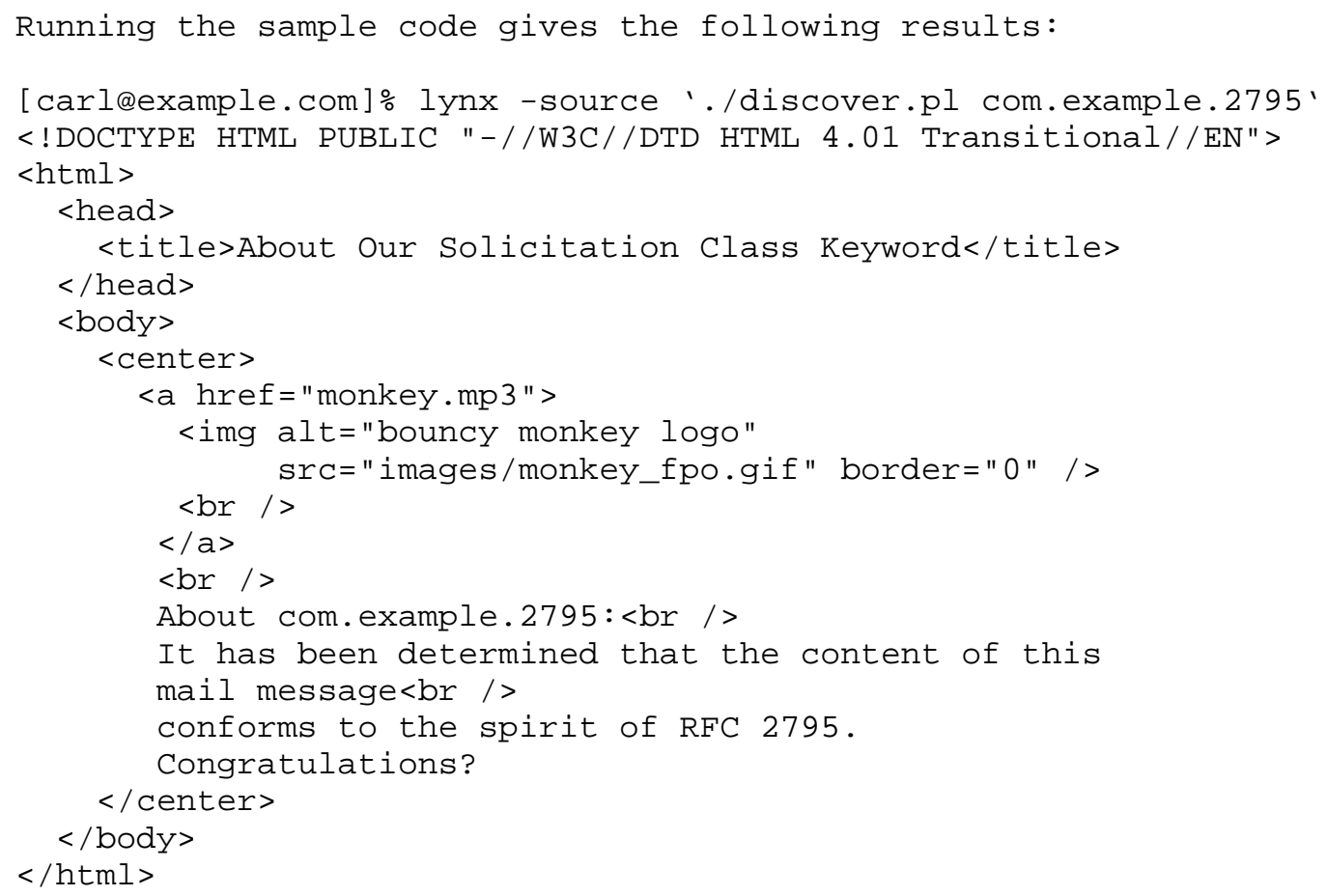

4. DDDS Application Specification

The following definitions apply to this application:

- Application Unique String: The application unique string is a Solicitation Class Keyword as defined in [RFC3865].

- First Well known Rule: The character "." is substituted for the character ":" and then the Solicitation Class Keyword is reversed in order to produce a valid domain name. For example, "com.example:adv" would become "adv.example.com".

- Valid Databases: The DNS _is_ the database.

o Expected Output: A URI.

o The "SERVICE" field MUST contain the string "no-solicit", the "FLAGS" field MUST contain the string "U", the "REPLACEMENT" field MUST be empty, and the "REGEXP" field MUST be formatted as specified in section 2 .

Wildcards are appropriate for this application, allowing multiple solicitation class keywords that share a common prefix to all point to the same URI. Note that the NAPTR Resource Record is known as a "subtyping" RR, which means that additional selectors are available within the RR to "winnow down" the choices. This means more records are returned than are actually needed, resulting in more traffic. 
But, this also means that wildcards may have unintended effects of multiple types of NAPTR resource records are used. Implementors and zone administrators should exercise care in the use of such wildcards in this application.

5. Acknowledgements

The author would like to thank the following for their helpful suggestions and reviews of this document: Leslie Daigle, spencer Dawkins, Arnt Gulbrandsen, Ted Hardie, Scott Hollenbeck, Russ Housley, David Kessens, Peter Koch, Michael Mealling, Pekka Savola, Mark Townsley, and Margaret Wasserman.

6. Security Considerations

This document specifies an application which depends on the Domain Name system to associate a solicitation class keyword with a URI. Four security considerations are raised by this application:

1. If the domain name lookup has been compromised, the application may return a URI with incorrect guidance on the use of a particular solicitation class keyword. In particular, if the application returns a URI with the "https:" scheme, and the DNS Security Extensions as defined in [RFC4033] and related documents are not used, the user would have an unwarranted illusion of authenticity making the possibility of active attacks a serious concern. Even if both DNS Security Extensions and the "https:" scheme are used, the client will need to take additional steps to ensure that the two different digital signature validation contexts are being administered by the same domain owner.

2. RFC 3865 bases solicitation class keywords on domain names. However, it does not define whom a user should trust. A sender or an intermediate MTA could insert a solicitation class keyword in a message and then use the application defined in this document to mislead the message recipient. For example, a malicious direct marketer might insert a keyword such as "org.example.certified.message" and use a URI to somehow indicate that the message (wrongly) has some official status. As with any URI, users must take further steps that are outside the scope of this specification to determine what and whom to believe.

3. Domain names are not persistent identifiers. As with any application that uses domain names, including the World Wide Web, if a domain name or a URI is embedded in an electronic mail message, there is a possibility that in the future the domain name will be controlled by a different zone administrator and that 
use of the application described in this document will yield different and possibly inconsistent results over time.

4. A malicious sender could insert a large number of solicitation class keywords or improperly formatted solicitation keywords, thus performing a Denial of Service attack on the recipient's resources through the use of an excessive number of DNS lookups. If such a message is sent to many recipients, this can result in a Denial of Service attack on the provider at a particular URI (e.g., a large number of requests attempting to access a URI such as "http://example.net/index.html"). Improperly formatted solicitation class keywords, particularly those with a nonexistent top level or second level domain, could result in a Denial of Service attack on DNS registry providers or the DNS root servers.

7. IANA Considerations

There is no central registry maintained by the IANA of values that might appear in the "SERVICE" field of a NAPTR resource record. Thus, no direct IANA actions are required.

However, the IANA does maintain an Application Service Tag Registry, which is used to support the S-NAPTR DDDS application defined in [RFC3958]. The IANA is advised that the "no-solicit" value for the SERVICE field is in use per this document and thus should not be used in the Application Service Tag Registry for other applications.

8. References

8.1. Normative References

[HTML-4.01] Raggett, D., Hors, A., and I. Jacobs, "HTML 4.01 Specification", W3C REC REC-html401-19991224, December 1999.

[RFC2119] Bradner, S., "Key words for use in RFCs to Indicate Requirement Levels", BCP 14, RFC 2119, March 1997.

[RFC2660] Rescorla, E. and A. Schiffman, "The Secure HyperText Transfer Protocol", RFC 2660, August 1999.

[RFC3402] Mealling, M., "Dynamic Delegation Discovery System (DDDS) Part Two: The Algorithm", RFC 3402, October 2002.

[RFC3403] Mealling, M., "Dynamic Delegation Discovery System (DDDS) Part Three: The Domain Name System (DNS) Database", RFC 3403, October 2002 . 
[RFC3865] Malamud, C., "A No Soliciting Simple Mail Transfer Protocol (SMTP) Service Extension", RFC 3865, September 2004 .

[RFC3958] Daigle, L. and A. Newton, "Domain-Based Application Service Location Using SRV RRS and the Dynamic Delegation Discovery Service (DDDS)", RFC 3958, January 2005.

[RFC3986] Berners-Lee, T., Fielding, R., and L. Masinter, "Uniform Resource Identifier (URI): Generic Syntax", STD 66, RFC 3986, January 2005 .

8.2. Informative References

[RFC2795] Christey, S., "The Infinite Monkey Protocol Suite (IMPS)", RFC 2795, 1 April 2000.

[RFC2821] Klensin, J., "Simple Mail Transfer Protocol", RFC 2821, April 2001 .

[RFC3401] Mealling, M., "Dynamic Delegation Discovery System (DDDS) Part One: The Comprehensive DDDS", RFC 3401, October 2002 .

[RFC3490] Faltstrom, P., Hoffman, P., and A. Costello, "Internationalizing Domain Names in Applications (IDNA)", RFC 3490, March 2003.

[RFC3761] Faltstrom, P. and M. Mealling, "The E.164 to Uniform Resource Identifiers (URI) Dynamic Delegation Discovery System (DDDS) Application (ENUM)", RFC 3761, April 2004.

[RFC4033] Arends, R., Austein, R., Larson, M., Massey, D., and S. Rose, "DNS Security Introduction and Requirements", RFC 4033, March 2005.

Author's Address

Carl Malamud

Memory Palace Press

PO Box 300

Sixes, OR 97476

US

EMail: carlemedia.org 
Full Copyright statement

Copyright (C) The Internet Society (2005).

This document is subject to the rights, licenses and restrictions contained in BCP 78, and except as set forth therein, the authors retain all their rights.

This document and the information contained herein are provided on an "AS IS" basis and THE CONTRIBUTOR, THE ORGANIZATION HE/SHE REPRESENTS OR IS SPONSORED BY (IF ANY), THE INTERNET SOCIETY AND THE INTERNET ENGINEERING TASK FORCE DISCLAIM ALL WARRANTIES, EXPRESS OR IMPLIED, INCLUDING BUT NOT LIMITED TO ANY WARRANTY THAT THE USE OF THE INFORMATION HEREIN WILL NOT INFRINGE ANY RIGHTS OR ANY IMPLIED WARRANTIES OF MERCHANTABILITY OR FITNESS FOR A PARTICULAR PURPOSE.

Intellectual Property

The IETF takes no position regarding the validity or scope of any Intellectual Property Rights or other rights that might be claimed to pertain to the implementation or use of the technology described in this document or the extent to which any license under such rights might or might not be available; nor does it represent that it has made any independent effort to identify any such rights. Information on the procedures with respect to rights in RFC documents can be found in BCP 78 and BCP 79 .

Copies of IPR disclosures made to the IETF Secretariat and any assurances of licenses to be made available, or the result of an attempt made to obtain a general license or permission for the use of such proprietary rights by implementers or users of this specification can be obtained from the IETF on-line IPR repository at http://www.ietf.org/ipr.

The IETF invites any interested party to bring to its attention any copyrights, patents or patent applications, or other proprietary rights that may cover technology that may be required to implement this standard. Please address the information to the IETF at ietfipreietf.org.

Acknowledgement

Funding for the RFC Editor function is currently provided by the Internet Society. 